\title{
AVALIAÇÃO DAS CONDIÇÕES DE CONSERVAÇÃO E LIMPEZA QUÍMICA PARA REUTILIZAÇÃO DE MEMBRANAS DE OSMOSE INVERSA DESCARTADAS DE PROCESSOS DE DESMINERALIZAÇÃO DE ÁGUAS
}

\author{
J. M. FRICK ${ }^{1}$, D. F. ROSA ${ }^{1}$, M. K. da $\operatorname{SILVA}^{1}$, L. D. POLLO ${ }^{1}$ e I. C.TESSARO ${ }^{1}$ \\ ${ }^{1}$ Universidade Federal do Rio Grande do Sul, Departamento de Engenharia Química \\ E-mails para contato: juliamfrick@gmail.com, isabel@enq.ufrgs.br
}

\begin{abstract}
RESUMO - O desempenho de membranas de osmose inversa descartadas para reutilização em outros processos de tratamento foi estudado neste trabalho. Foram avaliadas técnicas de limpeza química, conservação de membranas e dosagem de produtos químicos. Foram analisados três módulos espirais descartados de um sistema de desmineralização de águas: um sem conservação (A), um conservado com uma solução biocida (B) e um módulo retirado da unidade e imediatamente testado (C). O menor fluxo inicial $\left(0,1 \mathrm{~L} \cdot \mathrm{m}^{-2} \cdot \mathrm{h}^{-1}\right)$ foi atingido pelo módulo na condição (A) e o maior fluxo $\left(20,4 \mathrm{~L} \cdot \mathrm{m}^{-2} \cdot \mathrm{h}^{-1}\right)$ na condição $(\mathrm{C})$. A utilização de soluções de limpeza com ácido cítrico e hidróxido de sódio mostrou-se eficiente na remoção de incrustações e recuperação do fluxo permeado, alcançando $27,2 \mathrm{~L} \cdot \mathrm{m}^{-2} \cdot \mathrm{h}^{-1}$ no caso do módulo na condição C.
\end{abstract}

\section{INTRODUÇÃO}

A crescente escassez de água potável incentiva a busca por métodos de recuperação e tratamento de águas e efluentes ainda mais eficientes. A dessalinização é um dos tratamentos não convencionais mais utilizado em diversos países para a obtenção de água potável ou industrial, especialmente onde há escassez de água doce. Combinando estratégias de reutilização e a tecnologia de dessalinização, as águas residuais podem ser reaproveitadas em várias outras aplicações (Madwar e Tarazi, 2002).

A Osmose Inversa (OI) é um processo de separação por membranas bastante utilizado na dessalinização e desmineralização de águas, principalmente quando é necessária uma qualidade muito alta da corrente tratada. As membranas utilizadas na separação apresentam um tempo de vida útil de aproximadamente 3 a 5 anos, após o qual perdem a sua alta eficiência, sendo então substituídas, gerando grandes quantidades de módulos que são descartados e acabam sendo dispostos em aterros. Porém, estas membranas, mesmo não fornecendo a eficiência desejada para os sistemas de desmineralização, ainda poderiam ser reutilizadas em outros tratamentos, como por exemplo, em correntes de reuso, aumentando, desta forma, o tempo de vida útil destes módulos descartados e reduzindo a geração e o descarte de efluentes industriais (Rodríguez et al., 2002).

Pesquisas envolvendo a reutilização de módulos descartados são recentes e inovadoras, e 


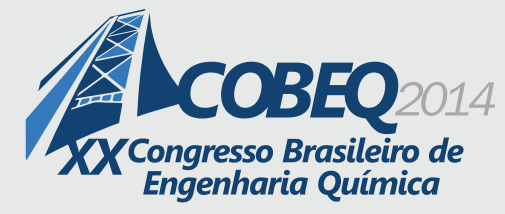

19 a 22 de outubro de 2014

Florianópolis/SC

poucas publicações são encontradas nesta área. Uma vasta gama de possibilidades pode ser considerada para a reutilização e reciclagem destas membranas, como a aplicação direta em sistemas de rendimentos mais baixos, reciclagem dos vários componentes do módulo, recuperação de energia através da incineração, e ainda modificações químicas para utilização em outros processos como ultrafiltração (UF) e microfiltração (MF) (Lawler et al., 2012). Como exemplo, Mohamedou e colaboradores (2010) estudaram membranas descartadas de OI, caracterizando-as através da permeabilidade e da retenção salina. Os autores observaram que a permeabilidade e a retenção salina destas membranas foram bem menores em comparação às membranas novas de OI, apresentando valores próximos às de nanofiltração $(\mathrm{NF})$, o que justifica a reutilização em outros processos de tratamento.

Outra possibilidade, além do reuso direto, seria a modificação das membranas através de um tratamento químico. Lawler et al. (2011) testaram a aplicação de diferentes agentes oxidantes para a degradação parcial ou total da camada seletiva, de modo a reutilizá-las em outros processos como, por exemplo, na UF. Ambrosi e Tessaro (2013) estudaram o tratamento químico de membranas de OI descartadas com soluções de permanganato de potássio em combinação com limpeza química, de modo a recuperar o fluxo de permeado e torná-las reutilizáveis, aumentando o seu ciclo de vida. Os resultados demonstraram que o permanganato de potássio é um agente eficaz para tornar a membrana reutilizável.

Segundo Rodrigues et al. (2003), um indicativo da possibilidade de reutilização de uma membrana é a medida da recuperação do fluxo de permeado após limpeza química. O método de limpeza depende do módulo, das características da membrana e da natureza do material depositado. As limpezas químicas removem os depósitos e as incrustações, além de evitar que novas substâncias ataquem a superfície da membrana (Hoof et al., 1999). Os principais fatores na escolha de um agente de limpeza são: capacidade de limpeza, custo, afinidade do agente químico com o material a ser removido, entre outros. Madaeni e Samieirad (2010) testaram uma variedade de produtos químicos, incluindo ácidos, bases, agente de complexação, surfactante e diferentes combinações destes produtos, para a remoção de incrustações de membranas de osmose inversa, sendo que a combinação base, surfactante e ácido proporcionou maior eficiência de limpeza.

Neste contexto, este trabalho se insere num projeto que visa estudar a possibilidade de reuso de módulos espirais de OI descartados de uma unidade de desmineralização de água, para reutilização no tratamento de correntes industriais. Os objetivos específicos apresentados neste artigo são o estudo da caracterização de módulos espirais descartados em diferentes condições de conservação e da eficácia de limpezas químicas na recuperação do fluxo de permeado e da retenção salina.

\section{MATERIAIS E MÉTODOS}

\subsection{Planta Piloto}

Os testes foram realizados em uma planta piloto localizada em uma empresa petroquímica no Rio Grande do Sul, Brasil. A planta é composta basicamente por um sistema de pré-tratamento, com filtro de areia por gravidade e filtro cartucho, tanques dosadores de produtos químicos, tanque de 


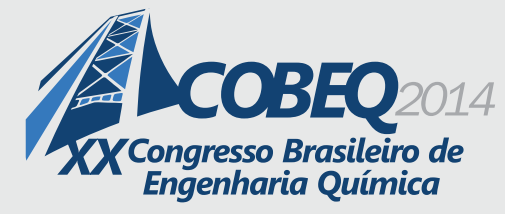

limpeza e vaso de pressão para o acondicionamento do módulo espiral com $20 \mathrm{~cm}$ de diâmetro e comprimento de $1,0 \mathrm{~m}$. Para prevenir incrustações e oxidação nas membranas e garantir o bom desempenho da unidade, é realizada a dosagem controlada de anti-incrustantes e agentes redutores. $\mathrm{O}$ tanque de limpeza apresenta duas finalidades, durante a operação é utilizado como tanque pulmão da unidade e durante a etapa de limpeza das membranas funciona como tanque de preparo e armazenamento de soluções.

A alimentação entra na unidade através do filtro de areia e, em seguida, Tripol ® (antiincrustante) e metabissulfito de sódio são dosados, no tanque de produtos químicos, para remoção de microrganismos, agentes oxidantes e cloro. Após, a alimentação passa pelo filtro cartucho, sendo então bombeada para o módulo de membrana espiral disposta no interior do vaso de pressão. Parte da corrente de concentrado é recirculada e parte é descartada como efluente orgânico. O permeado é descartado juntamente com a água da chuva. A temperatura e a condutividade elétrica são indicadas no painel de controle.

Para a limpeza química das membranas, as soluções são preparadas no tanque de limpeza com água filtrada, operando o sistema conforme descrito acima. As correntes de concentrado e permeado são recolhidas no tanque de alimentação em modo de reciclo total. A pressão para realização das limpezas deve ser baixa para não degradar as membranas; foi utilizada a pressão de 3,5 bar.

A caracterização das membranas foi realizada através dos parâmetros fluxo de permeado e retenção salina. Para estes testes, água filtrada foi utilizada como água de alimentação, com vazão de 12 gpm e pressão de 9 bar.

\subsection{Membranas Utilizadas}

As membranas utilizadas neste trabalho foram descartadas de um processo de desmineralização de águas, sendo que as características fornecidas pelo fabricante estão apresentadas na Tabela 1. Cabe salientar que as membranas testadas neste estudo operaram durante 3 anos em um sistema de desmineralização de águas, apresentando características de fluxo e retenção salina diferentes dos que constam na tabela.

Tabela 1 - Características das membranas de OI novas, fornecidas pelo fabricante

\begin{tabular}{lc}
\hline \multicolumn{2}{c}{ Dados das Membranas } \\
\hline Modelo & 8040 - ACM1 - TSA - Trisep \\
Material & Membrana Composta de Poliamida Aromática \\
Configuração & Módulo espiral, envolto em fibra de vidro \\
Área $\left(\mathbf{m}^{\mathbf{2}}\right)$ & 33,4 \\
Máxima vazão de alimentação $\left(\mathbf{m}^{\mathbf{3}} \cdot \mathbf{h}^{-\mathbf{1}}\right)$ & 18,0 \\
Faixa de pH da alimentação & $4-11$ \\
Faixa de temperatura de operação recomendada $\left({ }^{\circ} \mathbf{C}\right)$ & $2-45$ \\
Faixa de pressão recomendada (bar) & $7-21$ \\
Máximo valor de pressão suportado (bar) & 41 \\
Máximo SDI (15 minutos) & 5,0 \\
Máxima turbidez $(\mathbf{N T U})$ & 1,0 \\
Média de rejeição $(\% \mathbf{N a C l})$ & $>99,5$ \\
\hline
\end{tabular}




\subsection{Preservação das Membranas}

A influência da preservação das membranas no fluxo de permeado inicial e retenção salina foram avaliadas. De acordo com o tipo de armazenamento utilizado, os módulos de membranas foram classificadas como conservadas e sem conservação.

Os módulos sem conservação são aqueles armazenados sem qualquer cuidado, sem dosagem de produtos químicos para evitar o crescimento de microrganismos e estocagem em local aberto, sob a ação de intempéries, dispostos no pátio da empresa, durante dois anos (módulo A). O módulo de membrana conservada foi lavada com água filtrada e tratada com uma solução contendo um agente biocida (Kathon ${ }^{\circledR}$ WT), por 2 meses antes de seu uso, de modo a minimizar o crescimento de microrganismos (módulo B). Também foi avaliado um módulo diretamente recolhido do sistema de desmineralização e imediatamente inserido na planta piloto (módulo C).

\subsection{Limpezas Químicas}

As limpezas químicas foram realizadas como tentativa de recuperação do fluxo de permeado. Foram testadas limpezas combinadas de ácido/base. Segundo Oliveira (2007), a base mais utilizada para a limpeza das membranas é o hidróxido de sódio e o ácido mais usado é o cítrico, principalmente devido à sua capacidade de atuar como agente quelante.

Para a limpeza ácida foi utilizado ácido cítrico a 2\%; as membranas foram deixadas em contato com esta solução durante 30 minutos, em pH 4, com o principal objetivo de remover carbonatos. Para a limpeza básica foi usado hidróxido de sódio a $0,5 \%$, com circulação no sistema durante 30 minutos, em $\mathrm{pH} 11$, para a remoção, principalmente, de materiais orgânicos. Ainda, um teste utilizando metabissulfito de sódio foi realizado com o módulo A, a 1\%, durante 1 hora, como uma tentativa de promover o inchamento das cadeias poliméricas da membrana e aumentar o fluxo de permeado.

\section{RESULTADOS E DISCUSSÃO}

\subsection{Caracterização inicial dos fluxos de permeado}

A caracterização inicial das membranas selecionadas foi realizada para determinar a influência do tipo de armazenamento dos módulos sobre o fluxo de permeado inicial. De acordo com Tang et al. (2007) a caracterização das membranas tem um papel crucial para uma completa compreensão das propriedades físico-químicas das membranas de OI, seu desempenho, e a interação entre as membranas e os contaminantes presentes na alimentação, tais como materiais orgânicos e incrustações.

De acordo com a Figura 1, comparando-se o fluxo de permeado das membranas à pressão de 9 bar, o módulo A mostrou o menor fluxo de permeado inicial, devido provavelmente à secagem da membrana, por não ter sido armazenada com os devidos cuidados. O módulo $\mathrm{B}$, mostrou fluxo de permeado inicial de $17 \mathrm{~L} \cdot \mathrm{m}^{-2} \cdot \mathrm{h}^{-1}$. Por fim, o módulo $\mathrm{C}$, retirado da unidade de desmineralização e imediatamente realocado na planta piloto, apresentou o maior fluxo inicial, de $20,4 \mathrm{~L} \cdot \mathrm{m}^{-2} \cdot \mathrm{h}^{-1}$. Tal 


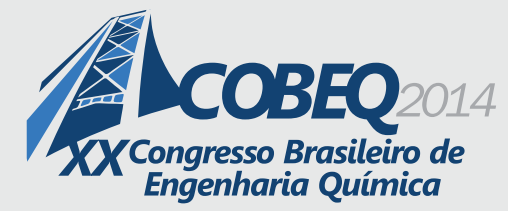

19 a 22 de outubro de 2014

Florianópolis/SC

comportamento pode ser explicado pelo fato de que, ao ser mantida em funcionamento, a membrana não passou por efeitos de secagem ou algum dano adicional devido ao contato com produtos químicos.

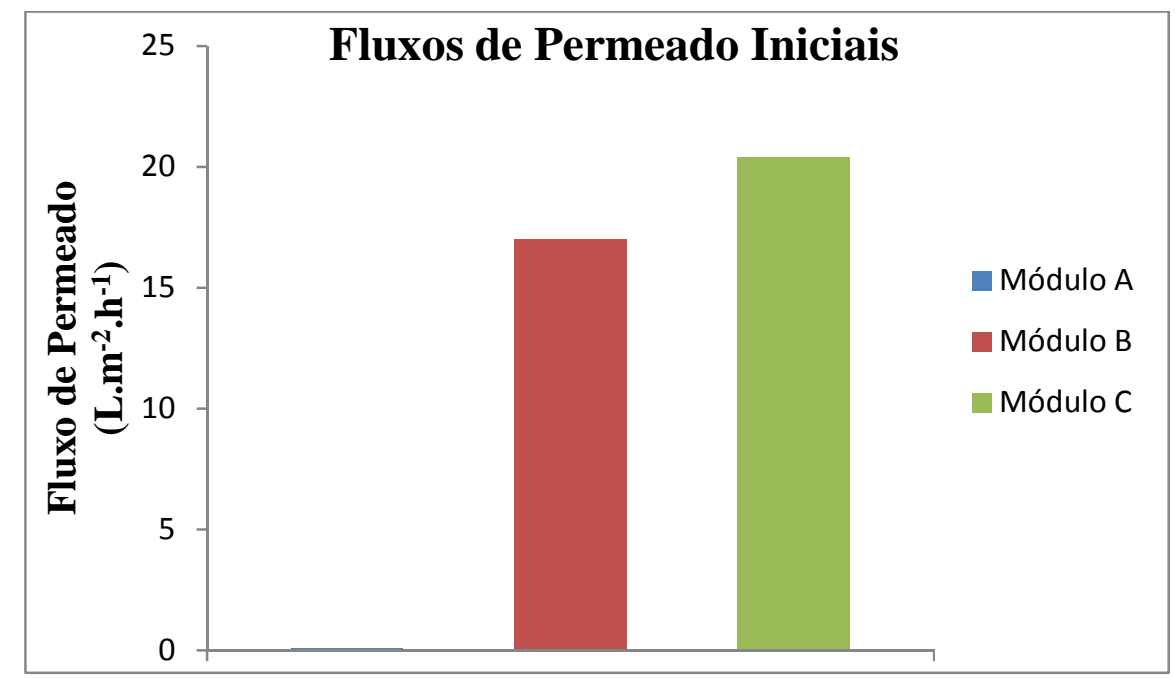

Figura 1 - Fluxos de permeado iniciais para os módulos A, B e C na pressão de 9 bar, vazão da corrente de alimentação de 12 gpm e temperatura ambiente $\left(25 \pm 2^{\circ} \mathrm{C}\right)$.

Módulos novos de membranas de osmose inversa, normalmente apresentam fluxo de permeado inicial de $37 \mathrm{~L} \cdot \mathrm{m}^{-2} \cdot \mathrm{h}^{-1}$, para uma pressão de 16 bar e uma permeância hidráulica igual a 2,3 L.m $\mathrm{m}^{-2} \cdot \mathrm{h}^{-1} \cdot \mathrm{bar}^{-1}$, tal como indicado pelo fabricante. Isto mostra que o fluxo de permeado inicial das membranas A e B foram inferiores aos de uma membrana nova. Para o módulo B a permeância inicial foi de $1,9 \mathrm{~L} \cdot \mathrm{m}^{-2} \cdot \mathrm{h}^{-1} \cdot \mathrm{bar}^{-1}$, já a membrana $\mathrm{C}$ apresentou o fluxo comparável com o de uma membrana nova, com permeância de $2,3 \mathrm{~L} \cdot \mathrm{m}^{-2} \cdot \mathrm{h}^{-1} \cdot$ bar $^{-1}$.

\subsection{Limpezas Químicas}

Nos experimentos de limpeza química das membranas, ácida e posteriormente básica, a pressão utilizada foi de 3,5 bar. Para o Módulo A, também foi realizada uma limpeza com metabissulfito de sódio. Cabe salientar que, após cada limpeza, foi feita nova caracterização das membranas em relação ao fluxo permeado para avaliação da influencia de cada etapa na recuperação do fluxo.

Na Figura 2 está representado o fluxo permeado após cada etapa de limpeza para o módulo A. Como pode ser observado, o fluxo de permeado aumentou para aproximadamente $11,6 \mathrm{~L} . \mathrm{m}^{-2} \cdot \mathrm{h}^{-1}$ após a limpeza ácida e foi alcançado um valor de $16,3 \mathrm{~L} \cdot \mathrm{m}^{-2} \cdot \mathrm{h}^{-1}$ após a limpeza alcalina. A limpeza com metabissulfito de sódio foi testada como uma técnica de modificação da superfície da membrana, visando o inchamento das cadeias poliméricas e o aumento do fluxo permeado, porém este processo não mostrou alteração do fluxo em relação às limpezas anteriores, sendo descartado dos experimentos seguintes. 


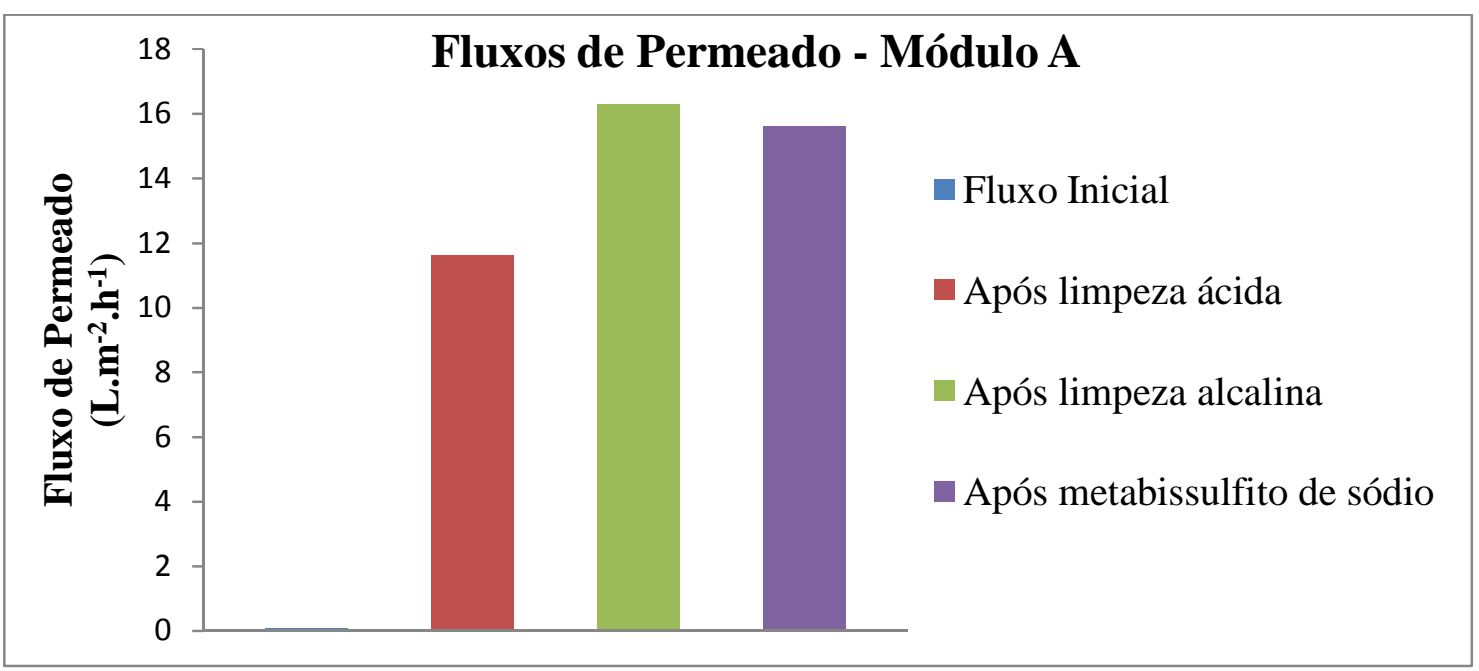

Figura 2 - Fluxos de permeado recuperados após as diferentes etapas de limpeza química para o Módulo A: vazão de $12 \mathrm{gpm}$; pressão de 9 bar e temperatura ambiente $\left(25 \pm 2^{\circ} \mathrm{C}\right)$.

A permeância hidráulica após a limpeza completa foi de $1,48 \mathrm{~L} \cdot \mathrm{m}^{-2} \cdot \mathrm{h}^{-1} \cdot \mathrm{bar}^{-1}$, representando uma recuperação de $64 \%$ do fluxo de permeado em comparação a uma membrana nova de OI. A retenção salina ficou em torno de $98 \%$ sendo que para membranas novas a retenção é de $99,5 \%$.

Para o módulo $\mathrm{B}$, os valores dos fluxos de permeado após cada limpeza estão representados na Figura 3. O fluxo inicial obtido foi $17 \mathrm{~L} \cdot \mathrm{m}^{-2} \cdot \mathrm{h}^{-1}$, alcançando-se um valor de $20,4 \mathrm{~L} \cdot \mathrm{m}^{-2} \cdot \mathrm{h}^{-1}$ após a limpeza alcalina. A permeância hidráulica para o módulo B foi igual a 2,27 L.m $\mathrm{m}^{-2} \cdot \mathrm{h}^{-1} \cdot \mathrm{bar}^{-1}$, equivalente ao valor apresentado pelas membranas novas. A retenção salina ficou em $97,7 \%$ para este módulo.

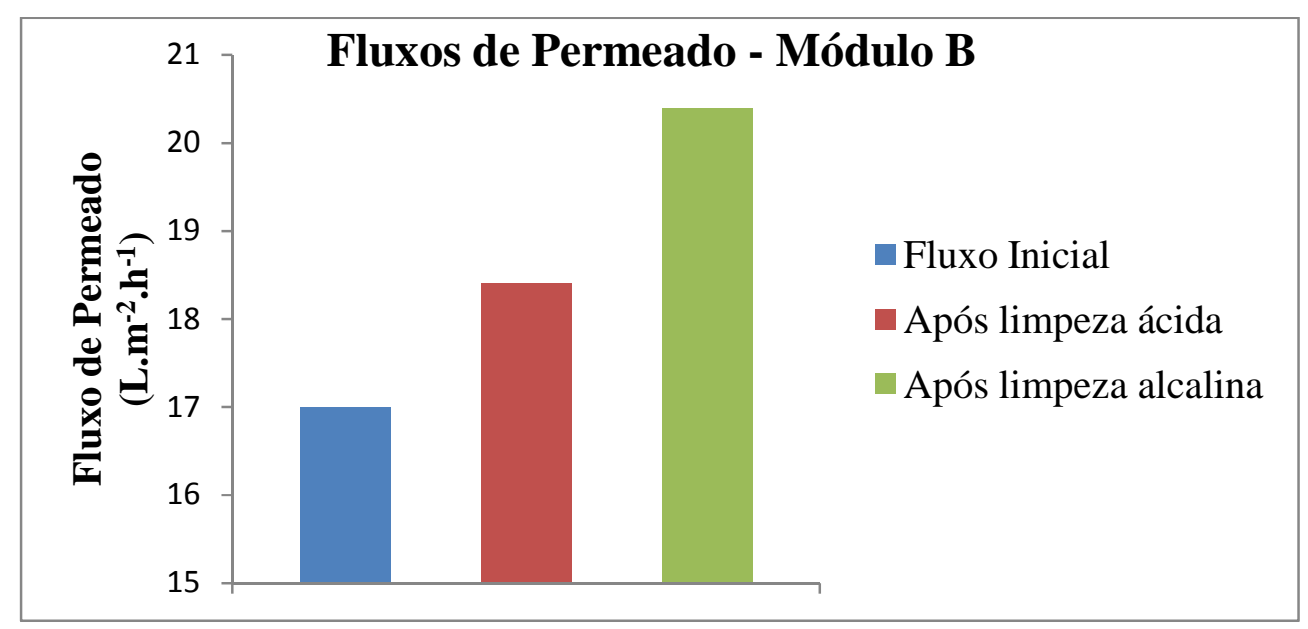

Figura 3 - Fluxos de permeado recuperados após as diferentes etapas de limpeza química para o Módulo B: vazão de $12 \mathrm{gpm}$; pressão de 9 bar e temperatura ambiente $\left(25 \pm 2^{\circ} \mathrm{C}\right)$. 


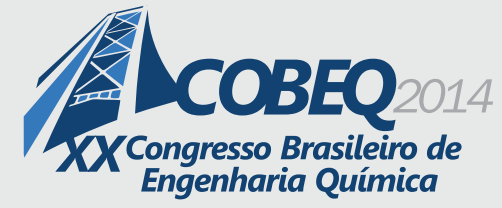

No módulo C, o fluxo de permeado inicial obtido foi 20,4 L.m $\mathrm{m}^{-2} \cdot \mathrm{h}^{-1}$. Analisando a Figura 4, após a limpeza ácida o fluxo foi recuperado para $23,8 \mathrm{~L} \cdot \mathrm{m}^{-2} \cdot \mathrm{h}^{-1}$, alcançando um valor de $27,2 \mathrm{~L} \cdot \mathrm{m}^{-2} \cdot \mathrm{h}^{-1}$ após a realização da limpeza alcalina. O valor da permeância hidráulica foi igual a $3 \mathrm{~L} \cdot \mathrm{m}^{-2} \cdot \mathrm{h}^{-1} \cdot \mathrm{bar}^{-1}$, valor próximo das membranas novas de OI. A retenção salina foi determinada, ficando em torno de $98,5 \%$ após a limpeza química.

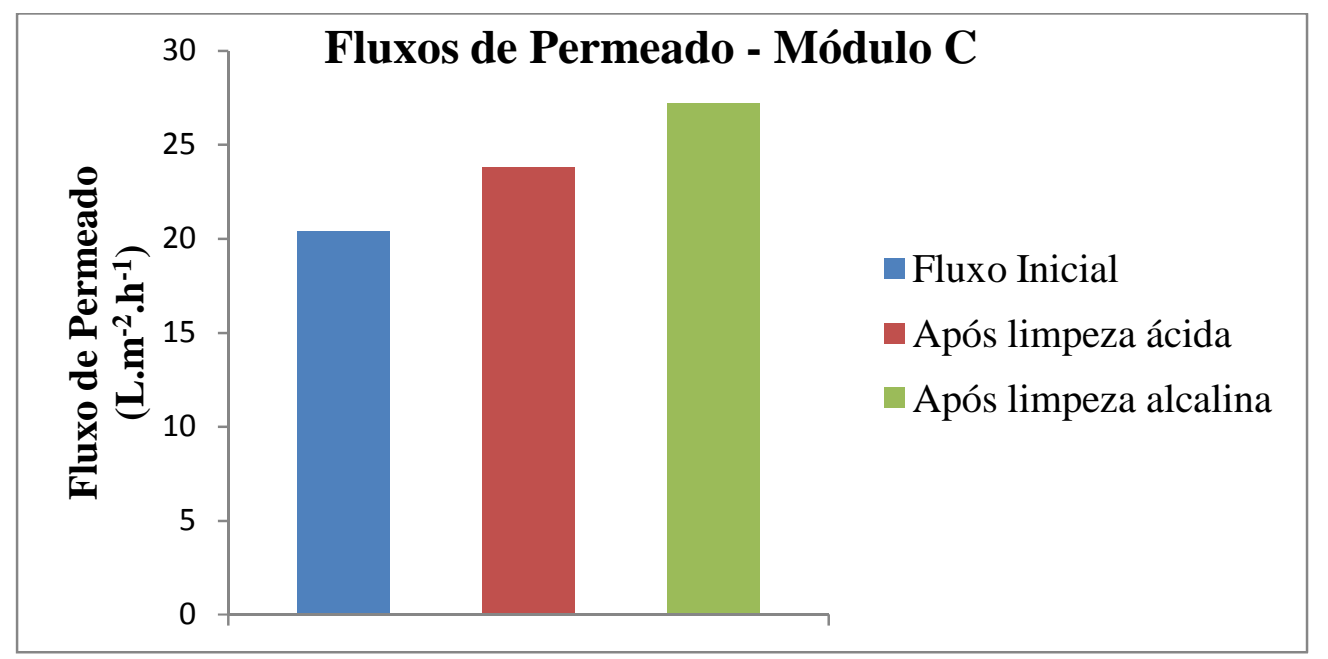

Figura 4 - Fluxos de permeado recuperados após as diferentes etapas de limpeza química para o Módulo C: vazão de $12 \mathrm{gpm}$; pressão de 9 bar e temperatura ambiente $\left(25 \pm 2^{\circ} \mathrm{C}\right)$.

No que diz respeito à limpeza química, verifica-se que o módulo $\mathrm{C}$ apresentou o melhor resultado para a recuperação do fluxo de permeado em relação ao fluxo permeado de uma membrana nova de OI, obtendo-se um valor de fluxo de permeado final igual a $27,2 \mathrm{~L} \cdot \mathrm{m}^{-2} \cdot \mathrm{h}^{-1}$, seguido do módulo B, com o fluxo de permeado de $20,4 \mathrm{~L} \cdot \mathrm{m}^{-2} \cdot \mathrm{h}^{-1}$ e o módulo A, com $16,3 \mathrm{~L} \cdot \mathrm{m}^{-2} \cdot \mathrm{h}^{-1}$.

\section{CONCLUSÕES}

O modo de conservação dos módulos de membranas tem grande influência nos valores dos fluxos iniciais de permeado e consequentemente na possibilidade de reuso das membranas. Observouse que, para o módulo sem conservação (A), o fluxo de permeado inicial foi muito baixo. O módulo $\mathrm{B}$, preservado em solução biocida, apresentou o fluxo de permeado inicial de $17 \mathrm{~L} \cdot \mathrm{m}^{-2} \cdot \mathrm{h}^{-1}$ e o módulo C, mantido em operação "quase" contínua, apresentou o maior fluxo de permeado inicial, igual a 20,4 L.m ${ }^{-2} \cdot h^{-1}$. De acordo com estes resultados, conclui-se que o ideal seria reutilizar o módulo logo após sua retirada do sistema de OI e, caso não sendo possível, conservá-lo em condições adequadas.

A limpeza química combinada ácido/base se mostrou eficiente para recuperação do fluxo de permeado das membranas. Os resultados ainda indicam a possibilidade de reutilização dos módulos dispostos em outros processos de tratamento, visto que os valores de fluxo permeado e retenção salina obtidos ao final das limpezas químicas foram satisfatórios. 


\section{REFERÊNCIAS}

AMBROSI, A.; TESSARO, I. C. Study on potassium permanganate chemical treatment of discarded reverse osmosis membranes aiming their reuse. Separation Science and Technology, v. 48, p. 15371543, 2013.

HOOF, S. C. J. M.; HASHIM, A.; KORDES, A. J. The effect of ultrafiltration as pretreatment to reverse osmosis in wastewater reuse and seawater desalination applications. Desalination, v. 124, p. 231-242, 1999.

LAWLER, W.; HARTKE, Z. B.; CRAN,M. J.; DUKE, M.; LESLIE, G.; LADEWIG, B. P.; CLECH, P. L. Towards new opportunities for reuse, recycling and disposal of used reverse osmosis membranes. Desalination, v. 299, p. 103-112, 2012.

LAWLER, W.; WIJAYA, T.; ANTONY, A.; LESLIE, G.; LE-CLECH, P. Reuse of reverse osmosis desalination membranes. IDA World Congress, 2011.

MADAENI, S. S; SAMIEIRAD, S. Chemical cleaning of reverse osmosis membranes fouled by wastewater. Desalination, v. 257, p. 80-86, 2010.

MADWAR, K.; TARAZI, H. Desalination techniques for industrial wastewater reuse. Desalination, v. 152, p. 325-332, 2002.

MOHAMEDOU, E. O.; SUAREZ, D. B.; VINCE, F.; JAOUEN, P.; PONTIE, M. New lives for old reverse osmosis (RO) membranes. Desalination, v. 253, p. 62-70, 2010.

OLIVEIRA, D. R. Pré-tratamento do processo de osmose inversa utilizando microfiltração e investigação de técnicas de limpeza e recuperação de membranas. Rio de Janeiro: COPPE, 2007. Dissertação de Mestrado, Faculdade de Engenharia Química, Universidade Federal do Rio de Janeiro, 2007.

RODRIGUES, S. L. C.; MOREIRA, R. L. S.; CARDOSO, M. H.; MERÇON, F. Avaliação de parâmetros de ultrafiltração de suco de banana. Ciência e Tecnologia de Alimentos, v. 23, p. 98-101, 2003.

RODRÍGUEZ, J. J.; JIMÉNEZ, V.; TRUJILLO, O.; VEZA, J. M. Reuse of reverse osmosis membranes in advanced wastewater treatment. Desalination, v. 150, p. 219-225, 2002.

TANG, C. Y.; KWON, Y. N.; LECKIE, J. O. Probing the nano- and micro-scales of reverse osmosis membranes - A comprehensive characterization of physiochemical properties of uncoated and coated membranes by XPS, TEM, ATR-FTIR, and streaming potential measurements. Journal of Membrane Science, v.287, p. 146-156, 2007. 